\title{
The effect of age on the dynamics and the level of c-Fos activation in response to acute restraint in Lewis rats
}

\author{
Ksenia Z. Meyza, Pawel M. Boguszewski, Evgeni Nikolaev, Jolanta Zagrodzka* \\ Nencki Institute of Experimental Biology, Polish Academy of Sciences, 3 Pasteur Street, 02-093 Warsaw, Poland \\ Received 7 November 2006; received in revised form 13 February 2007; accepted 12 March 2007 \\ Available online 14 March 2007
}

\begin{abstract}
Recent studies have reported an age-related increase of anxiety in rodents with a concomitant decrease in neuronal activity in some of the key structures of the fear/anxiety circuit. In the present study we present evidence that distinct parts of this circuit are differentially affected by age in Lewis rats. The effect of ageing is observed both at the actual level of neuronal activation and its time-course. While the structures belonging to the HPA axis react with a bigger neuronal activation and almost no change in the shape of dynamics curve in response to restraint, the structures involved in higher processing of emotional cues (amygdala and hippocampus) become deficiently activated with age despite their generally higher basal level of activation.
\end{abstract}

(C) 2007 Published by Elsevier B.V.

Keywords: Stress; Ageing; HPA axis; Amygdala; Hippocampus; c-Fos protein; Lewis rats

\section{Introduction}

Since the discovery of c-Fos as a functional marker of neuronal activation, expression of this protein has been used in many studies to identify cells and specific brain circuits that are responsive to various stimuli $[23,31,35]$. With this method it has been demonstrated that an increase in anxiety is associated with increased c-Fos expression in specific brain areas implicated in the regulation of emotional behavior including the amygdala, hypothalamus and hippocampus [8,9,22,29,31,35].

The anxiety level is known to be affected by age in humans as well as in animals. In recent years several studies have reported increased anxiety with age in rodents using a variety of wellvalidated tests of anxiety $[4,6,17,24-28]$. It could therefore be hypothesized that the increased anxiety in older rats should be associated with elevated neuronal activation in key parts of the fear/anxiety circuit. To the best of our knowledge, only a very few papers have directly addressed this question $[6,34]$. Salchner et al. [34] found that in spite of an age-related reduction of social interaction, indicative of increased anxiety [16], old rats exhibited a lower test-induced c-Fos response than young rats in the amygdala, periaqueductal grey and paraventricular hypotha-

\footnotetext{
* Corresponding author. Tel.: +48 225892246; fax: +48 228225342.

E-mail address: j.zagrodzka@nencki.gov.pl (J. Zagrodzka).
}

lamic nucleus. Based on this finding, the authors suggested that reduced social interaction does not reflect enhanced anxiety in old rats. Similar results were described by Boguszewski and Zagrodzka [5]. In this preliminary study c-Fos expression in the amygdala was significantly lower in old rats compared with young rats after the open field test and immobilisation, despite demonstrating an increased level of anxiety as measured in a set of standard laboratory tests and evaluated by principal component analysis [6]. One of the possible explanations of these somewhat unexpected results points to age-related differences in dynamics of c-Fos production. Most comparative studies of gene expression during aging have been conducted at a single time point usually matched to the maximum mRNA or protein concentration in young animals. Studies of the dynamic curve of $c$-fos mRNA content as a function of time after pentylenetetrazole (PTZ)-induced seizures have shown, that maximum $c$-fos concentration in the hippocampus and cortex of 3-month-old rats occurred $1 \mathrm{~h}$ post injection and $3 \mathrm{~h}$ later returned to basal levels. In contrast, in old rats (20 and 30 months old) there was a gradual increase in the levels of $c$-fos mRNA content, which reached a maximum $3 \mathrm{~h}$ after PTZ administration and returned to basal levels by approximately $15 \mathrm{~h}[33,36,42]$.

The aim of the present experiment is to compare the timecourse of c-Fos protein expression in response to acute restraint in young and old rats. Acute restraint is an extremely stressful 
stimulus known to evoke robust and widespread $c$-fos expression in numerous stress-specific and anxiety related structures [9].

\section{Material and methods}

Twelve young (YA, 3 months old) and 12 old (OA, 21-23 months old) male Lewis rats (from a breeding colony established at the Medical Academy, Warsaw, Poland) were housed in groups of two to three rats per cage (control animals housed separately from the experimental ones), with unlimited access to water and standard laboratory rat chow, in L:D 12:12 conditions, with lights on at 7:00 a.m. At the age of 3 and 21-23 months, respectively, the rats were subjected to acute restraint, for a duration of $15 \mathrm{~min}$, in a clear Plexiglass ventilated tube, $20 \mathrm{~cm}$ long, $6.5 \mathrm{~cm}$ inner diameter, with adjustable length according to the size of the animal and tails protruding. The size of the tube restricted movements in all directions but did not interfere with respiration. After the acute restraint procedure, rats were returned to their home cages. The rats (three animals per time point) were sacrificed with an overdose of chloride hydrate anaesthesia $(>360 \mathrm{mg} / \mathrm{kg}$ ) at $90 \mathrm{~min}, 150 \mathrm{~min}$ or $240 \mathrm{~min}$ after the beginning of the test and perfused transcardially with ice-cold phosphate buffered saline (PBS, pH 7.4 Sigma) followed by $4 \%$ paraformaldehyde ( $\mathrm{POCh}$ ) solution. Control animals (three animals per age group) were sacrificed directly from their home cages. Their brains were dissected and postfixed in $4 \%$ paraformaldehyde solution overnight and thereafter in $20 \%$ and $30 \%$ sucrose (Sigma) solutions. The brains were deep frozen and stored at $-72{ }^{\circ} \mathrm{C}$ until the day of sectioning in the cryostat $\left(-21^{\circ} \mathrm{C}\right)$. Forty-micrometer thick coronal sections were taken and subjected to standard c-Fos immunocytochemistry according to the procedure described in detail before [35].

c-Fos stained brain slices were microphotographed and bilaterally assessed for c-Fos activation using ImageJ software (WCIF, Toronto, Canada) in the amygdaloid complex, including basolateral (BLA), central (CeA), medial (MeA) and cortical (CoA) nuclei, the hippocampus (CA1, CA2 and CA3 fields) and in the hypothalamus including the paraventricular (PVN), dorsomedial (DMH) and arcuate (Arc) nuclei. Each structure was assesed on the basis of measures from three neighboring brain slices. For each brain structure, the number of
c-Fos immunopositive nuclei was counted and divided by the area occupied by this structure (in arbitrary units). The subnuclear division was obtained by comparison with the adjacent, Nissl-stained sections. Slices from the brains of three OA animals had to be excluded from the study post mortem due to the occurrence of tumors in the hypothalamus (one animal from the control group and one from $150 \mathrm{~min}$ ) and in the olfactory bulbs (one animal from $240 \mathrm{~min}$ group).

The study was conducted in accordance with the Polish Law on Animal Protection and the guidelines established by the Declaration of Helsinki concerning the care and use of animal in research.

Statistical analysis was performed using MANOVA and subsequent post-hoc Fischer/NIR tests. Values were considered significant if $p<0.05$.

\section{Results}

In this study, a structure specific difference in the dynamics of neuronal activation induced by acute restraint stress in young adult (YA) as compared with old (OA) rats was observed. The differences in relative activation (based on comparison of exact counts of c-Fos positive nuclei at a given time point with baseline activation) were observed between the two age groups at all experimental time points (90, 150 and $240 \mathrm{~min})$. The effect of age was significant $(F(1,32)=382.35, p<0.001)$. So was the effect of time points $(F(3,32)=14.21, p<0.001)$. The interaction of age and time points occurred to be structure specific $(F(27,288)=5.57, p<0.001)$.

The post-hoc analysis revealed that the time-course of neuronal activation in response to acute restraint in structures of the hypothalamic-pituitary-adrenocortical (HPA) axis (the paraventricular, arcuate and dorsomedial nuclei of the hypothalamus)
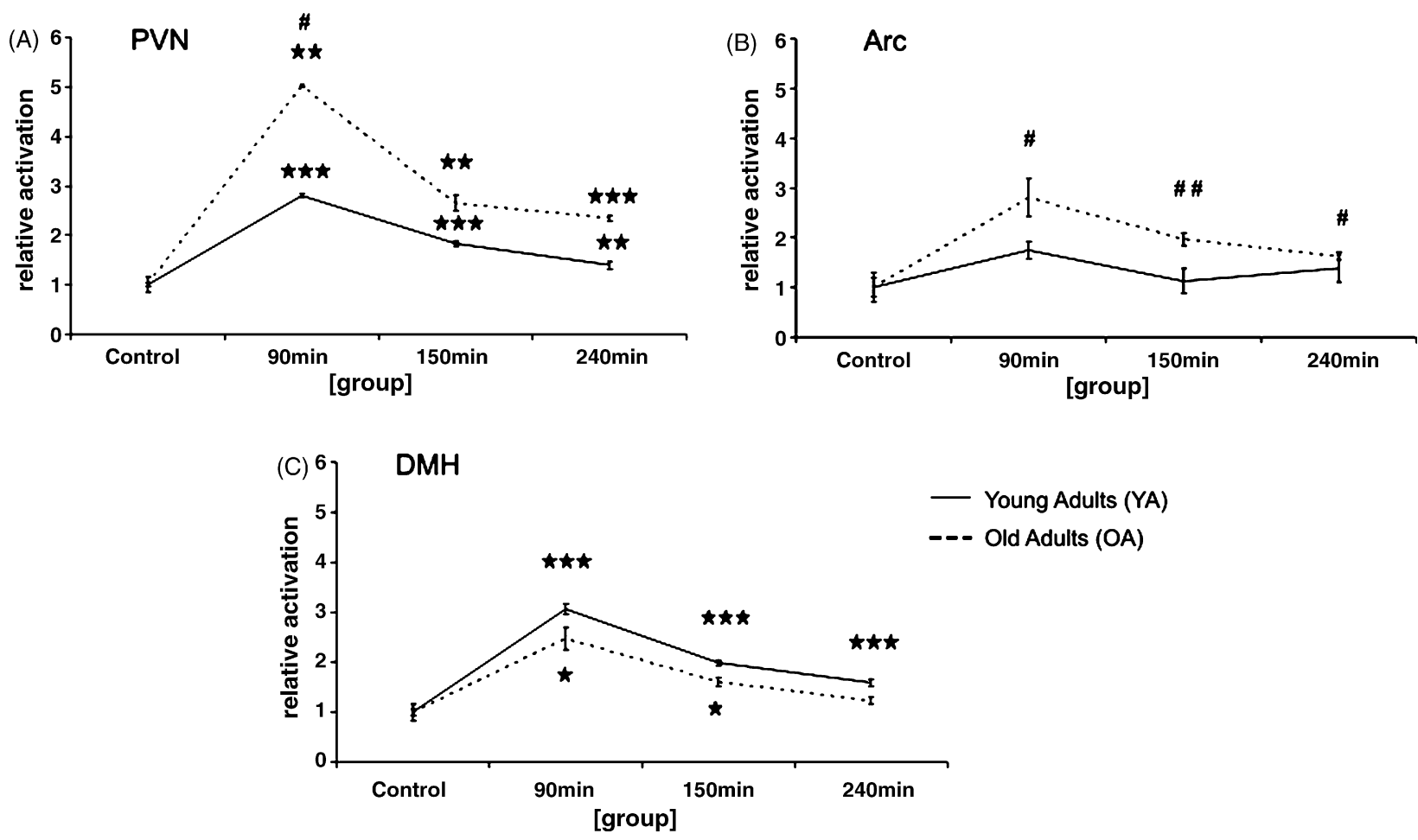

Fig. 1. The dynamics of neuronal activation assessed by c-Fos expression in (A) paraventricular (PVN), (B) arcuate (Arc), and (C) dorsomedial nuclei of the hypothalamus $(\mathrm{DMH})$ at $90 \mathrm{~min}, 150 \mathrm{~min}$ and $240 \mathrm{~min}$ after the onset of acute restraint. Continuous line represents YA. Broken line represents OA. Values are presented as mean \pm S.E.M. ${ }^{\#} p<0.05,{ }^{\# \#} p<0.01$ for comparison between YA and OA; ${ }^{*} p<0.05,{ }^{* *} p<0.01,{ }^{* * *} p<0.001$ for comparison with baseline within one age group. 
was unaffected by age. The highest neuronal activation was observed 90 min after the onset of stress in all three structures (Fig. 1). In the PVN the shape of the two curves was similar, although in old rats the increase in c-Fos expression was more profound than in young rats (Fig. 1A, $p<0.05$ ). We observed a five-fold increase in neuronal activation $90 \mathrm{~min}$ after stress in old rats $(p<0.001)$, while the peak value seen at the same time point in young rats reached only 2.8 times baseline (Fig. $1 \mathrm{~A}, p<0.01$ ). A similar, though less profound effect of age was found in the arcuate nuclei (Fig. 1B, $p<0.05$ ).

The temporal dynamics and the level of c-Fos expression in the dorsomedial nuclei of the hypothalamus were similar in OA and YA groups. The nuclei remained significantly activated at all time points in YA and $90 \mathrm{~min}$ and $150 \mathrm{~min}$ time points in OA group and there were no significant differences between the two groups (Fig. 1C).

In the central nuclei of the amygdala the maximum number of c-Fos positive neurons was found at different time points in YA and OA rats. In YA peak activation was observed 150 min after the onset of acute restraint $(p<0.01)$, while in OA c-Fos expression did not reach significance. Nevertheless a biphasic trend was observed with the first "peak" of activation $(p<0.05) 90 \mathrm{~min}$ after stress, and a second one at $240 \mathrm{~min}$ time point (Fig. 2A). No such difference in dynamics of c-Fos expression was observed in the basolateral, medial or cortical nuclei of the amygdala as the peak expression in YA and the maximum of the trend curve of OA occurred at 90 min time point (Fig. 2B-D). The peak of maximal c-Fos expression in OA did not reach significance due to an overall decrease in activation (as compared to baseline conditions) of all examined amygdalar nuclei in response to stress. This effect was observed despite the higher basal activation in OA (Fig. 4).

A significant secondary wave of activation was observed in 240 min time point in CoA (Fig. 2D) of young rats. No significant secondary activation was observed in aged rats.

Another significant difference in c-Fos protein expression dynamics was observed in the hippocampi (especially the CA1, CA2 fields). In YA a clear peak of c-Fos activation was observed in these fields $90 \mathrm{~min}$ after acute restraint $(p<0.001)$, after which the number of c-Fos positive nuclei slowly decreased. In contrast, in OA the number of c-Fos positive nuclei progressively increased up to $240 \mathrm{~min}$ after stress but still did not reach significance, when compared to the baseline values (Fig. 3A-C). The CA3 hippocampal field is different in this respect. A general lack of significant (as compared with baseline) activation was observed both in YA and OA. The only significant increase in the number of c-Fos positive nuclei was found in OA between $150 \mathrm{~min}$ and $240 \mathrm{~min}$ time points (Fig. 3C).

It is noteworthy that within the hypothalamus PVN and Arc are similarly affected by age. A similar, homogenous (though reverse to hypothalamic increase in activation with age) effect was observed for the CA1 and CA2 fields of hippocampi. On the other hand, the nuclei of amygdala represent a heterogeneous pattern. When young rats are challenged with an acute restraint
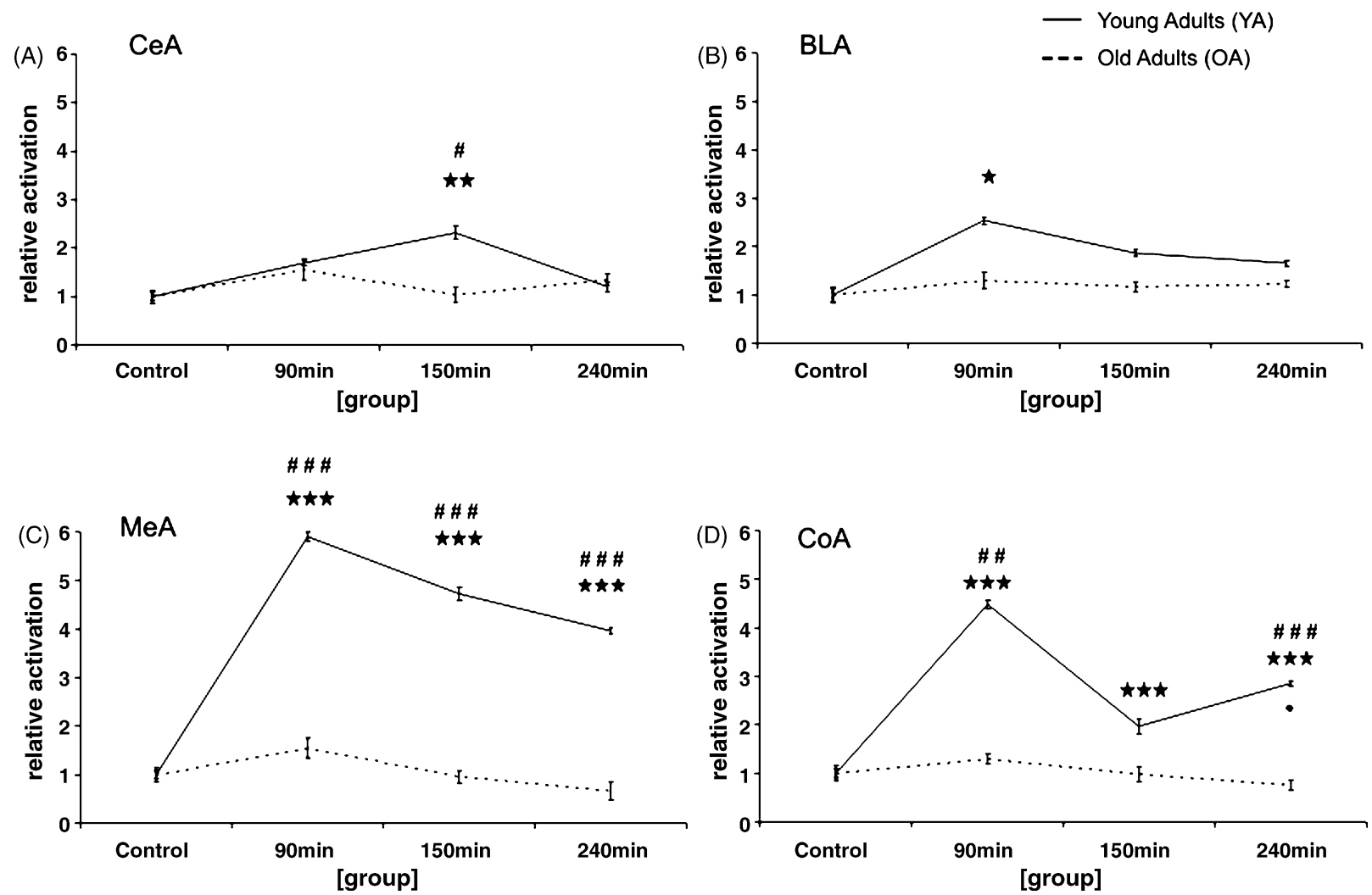

Fig. 2. The dynamics of neuronal activation assessed by c-Fos expression in (A) central (CeA), (B) basolateral (BLA), (C) medial (MeA) and (D) cortical nuclei of the amygdala $(\mathrm{CoA})$ at $90 \mathrm{~min}, 150 \mathrm{~min}$ and $240 \mathrm{~min}$ after the onset of acute restraint. Continuous line represents YA. Broken line represents OA. Values are presented as mean \pm S.E.M. ${ }^{\#} p<0.05,{ }^{\# \#} p<0.01,{ }^{\# \# \#} p<0.001$ for comparison between YA and OA; ${ }^{*} p<0.05, * * p<0.01, * * * p<0.001$ for comparison with baseline within one age group. $\bullet$ indicates $p<0.05$ for $150 \mathrm{~min}$ vs. $240 \mathrm{~min}$ secondary activation in CoA of YA. 

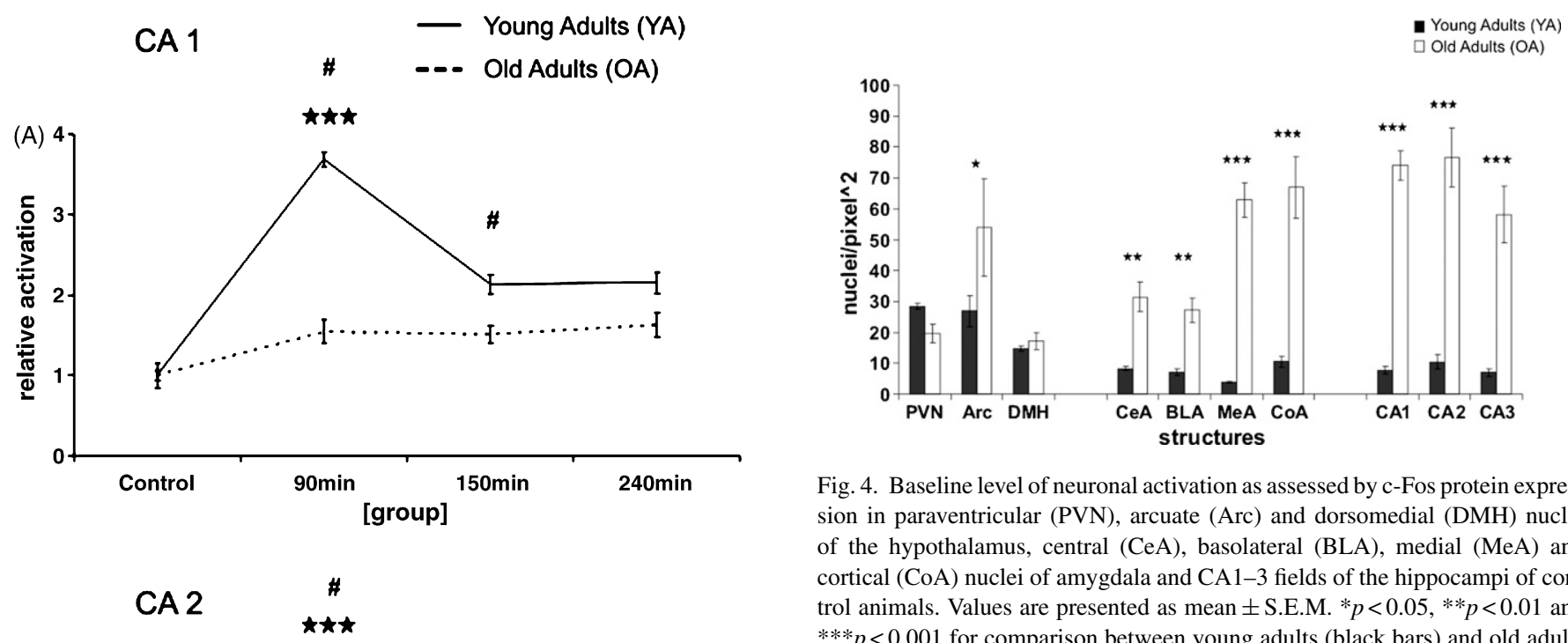

Fig. 4. Baseline level of neuronal activation as assessed by c-Fos protein expression in paraventricular $(\mathrm{PVN})$, arcuate $(\mathrm{Arc})$ and dorsomedial $(\mathrm{DMH})$ nuclei of the hypothalamus, central (CeA), basolateral (BLA), medial (MeA) and cortical (CoA) nuclei of amygdala and CA1-3 fields of the hippocampi of control animals. Values are presented as mean \pm S.E.M. ${ }^{*} p<0.05, * * p<0.01$ and $* * * p<0.001$ for comparison between young adults (black bars) and old adults (white bars)

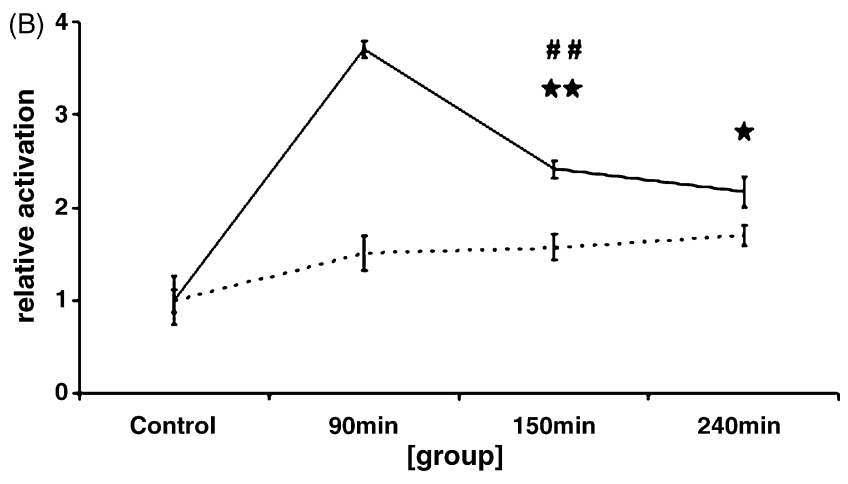

Notably the number of residual c-Fos positive nuclei is affected by age in amygdala and hippocampi (significantly increased in OA), while the basal activation of hypothalamic nuclei remains unchanged (except for the arcuate nuclei, where one individual had high residual c-Fos level, Fig. 4).

\section{Discussion}

We have shown that age substantially affects the level and to smaller extent the temporal dynamics of neuronal activation in key structures of the stress-response circuit, assessed by c-Fos immunoreactivity, in response to acute restraint. We observed a decreased neuronal responsiveness of the hippocampus and amygdala in old rats, suggesting that structures involved in higher processing of emotional stimuli may become deficient with age. In contrast, the neuronal activation of the PVN and Arc of the HPA axis, providing the neuroendocrine response to stress, was higher in old rats. At the same time the temporal dynamics of the response remained unchanged with age. Together, these observations support and add to previously reported data on the effect of age on neuronal activation following different types of stressful stimuli $[5,28,34]$.

Changes in endocrine function of HPA axis of aged Lewis

Fig. 3. The dynamics of neuronal activation assessed by c-Fos expression in (A) CA1, (B) CA2, and (C) CA3 fields of the hippocampi at $90 \mathrm{~min}, 150 \mathrm{~min}$ and $240 \mathrm{~min}$ after the onset of acute restraint. Continuous line represents YA. Broken line represents OA. Values are presented as mean \pm S.E.M. ${ }^{\#} p<0.05,{ }^{\# \#} p<0.01$, for comparison between YA and OA; $* p<0.05,{ }^{* *} p<0.01, * * * p<0.001$ for comparison with baseline within one age group. indicates $p<0.05$ for $150 \mathrm{~min}$ vs. 240 min secondary activation in CA3 of OA.

test, the medial and cortical nuclei of amygdala display the greatest increase in c-Fos immunoreactivity $90 \mathrm{~min}$ after the onset of acute restraint. The basolateral nuclei respond with intermediate values, while the central nuclei are the least activated. In aged rats subjected to the same stress, the activation in the central nuclei is the highest, followed by medial, basolateral and finally cortical nuclei. rats have been reported before [38]. The authors have found that the differences between Lewis and Fischer (F344) rats in corticosterone response to restraint diminished with age. Young Lewis rats of both sexes showed much smaller activation of HPA axis than F344 individuals. Eighteen-month-old male Lewis rats responded with similar to F344 males rise in corticosterone levels after stress, while Lewis female response was even greater than the one of F344 females. This is in accordance with our data on the increase in neuronal activation of PVN and Arc after acute restraint with age.

The dynamics of neuronal activation can be assessed in terms of the time-course of the maximal activation of a given structure and the shape of the dynamics curve. The latter reflects both the 
rate of activation and the actual levels of activation at a particular moment.

The time course of c-Fos expression in the paraventricular, dorsomedial and arcuate nuclei of the hypothalamus is very similar in both age groups. PVN and DMH responded with maximal c-Fos expression $90 \mathrm{~min}$ after the onset of acute restraint stress. So did the Arc nuclei but to a smaller extent. The shape of the dynamics curve in these structures is nevertheless different. We observed a more dramatic response of PVN and Arc neurons in old rats than in young rats. The dorsomedial nuclei on the other hand, did not exhibit any significant, age-dependent changes in the shape of the neuronal activation dynamics curve. One would expect that, instead, an increase in neuronal activation would occur in response to acute restraint, as has been reported previously $[8,9,23]$ in Sprague-Dawley, Lister Hooded and Wistar rats. This could be partially explained by the differences between the strains. Lewis rats are known for their reduced responsiveness to negative feedback loop of ACTH and CRH [19] in the HPA axis. Our results might indicate that dorsomedial nuclei are functionally deficient in old Lewis rats. If so, then the increase in maximal neuronal activation of the PVN and Arc might result from release of inhibitory influences of dorsomedial nuclei of hypothalamus [7,18,20,39].

In contrast to the hypothalamus, the pattern of neuronal activation following acute restraint is different in the central nuclei of amygdala. We observed an age-dependent change in the timecourse of maximal activation of these nuclei. In young animals the maximum activation occurred $150 \mathrm{~min}$ after the onset of acute restraint, while in aged rats the peak value (though still insignificant) was obtained $90 \mathrm{~min}$ after stress. The late peak of activation observed in our experiment in young animals is in contrast to results from a study using capsaicin [21], where a positive correlation between time and neuronal activation in the central nuclei of amygdala and paraventricular nuclei of the hypothalamus was found. This discrepancy might be due to the different nature of the stimulus, restraint being a processive stimulus [15] and capsaicin representing a systemic challenge. Alternatively this may be due to the fact the study was performed on Sprague-Dawley rats while Lewis rats were used for our experiment and these strains are believed to react differently to stressors $[32,38,41]$. The fact that a profound difference in the dynamics of neuronal activation was observed only in the central nuclei of young and old rats and not in the medial, cortical or basolateral nuclei of amygdala seems to be important and might implicate a different role of these nuclei in processing of aversive stimuli. Concomitantly and due to the difference in the time-course of maximal activation, we found a general quantitative difference in the level of activation of the nuclei of amygdala. In young animals the central nuclei are the least activated part of the amygdala (with medial nuclei being the most activated, followed by cortical and basolateral nuclei) at $90 \mathrm{~min}$ after stress. In aged rats the central nuclei are the most activated compared with the medial, basolateral and finally cortical nuclei. While the small activation rate of central amygdala at $1-2 \mathrm{~h}$ after stress had been reported before in young animals subjected to restraint $[3,11,12,23]$, our study is to the best of our knowledge the first one to show that this effect could be altered by age. Further studies are needed in order to elucidate whether this is due to an impairment of inhibitory action of lateral part of central amygdala or oval nucleus of bed nucleus of the stria terminalis as suggested by Day et al. $[10,11]$.

An age-dependent change in the dynamics of neuronal activation in response to acute restraint was observed in the hippocampus as well. The ability of the hippocampus to respond with neuronal activation to emotional stimuli has been considered controversial for many years. Some authors report no significant changes in neuronal activation after behavioral challenge $[13,34]$ while others postulate its role in control of emotions but with no specificity as to what kind of stressor is used [15]. It was also suggested that the hippocampus should only react to environmental cues encouraging exploratory behavior [30,37]. Nagahara and Handa suggested that even if the hippocampus reacts to stressful stimuli this change remains unaffected by age [28], while Touzani et al. [40] as well as Schmoll et al. [36] proved the opposite in experiments on mice and rats. Some authors suggest that only the CA1 field of the hippocampus exhibits a rise in c-Fos expression under conditions of restraint [9] while Kovacs suggests that activation is generalized onto other parts of this structure as well [23]. In our study we observed changes in the dynamics of c-Fos protein expression in CA1-2 hippocampal fields. In young animals these parts of the hippocampus are maximally activated 90 min after the onset of the acute restraint, with a subsequent decrease in activation at $150 \mathrm{~min}$ and $240 \mathrm{~min}$ time points. In aged rats the peak value per se does not exist and the activation rate steadily grows in all hippocampal fields achieving highest values $240 \mathrm{~min}$ after the onset of stress. This is in accordance with studies by Schmoll et al. [36] and Wagner et al. [42] who reported a shift into longer latencies of neuronal activation after seizures in the hippocampus and cortex of aged rats. They also found that the maximal neuronal activation in old rats was significantly smaller than the ones of young animals. Our study confirms this finding in the acute restraint stress model. One could argue that neuronal activation in the hippocampus might not be a direct result of restraint, but rather of the extensive exploration occurring after the animal is released back to its home cage. We cannot exclude that possibility as we did not record the behavior of the rats upon their return to home cages, but since such activation has been reported before $[9,23]$ we interpret our data as age-related differences in neuronal activation caused by acute restraint.

Apart from age-dependent differences in the time-course of maximal neuronal activation in structures responsible for higher processing of emotional stimuli (such as amygdala and hippocampi) as compared with the nuclei of the HPA axis we observed differences in respect to the shape of the decreasing slope of the dynamics curve in the cortical nuclei of amygdala. In the CoA of young rats a significant secondary increase in neuronal activation occurred $240 \mathrm{~min}$ after the onset of stress. Such late activation was observed for other immediate early gene, the Egr-1 (Zif-268) in response to acute restraint [9] which could implicate that some types of emotional information processing, possibly also involving c-Fos, might take place even up to $4 \mathrm{~h}$ after acute stress. 
Another observation made in this study was that c-Fos protein expression reaches significantly higher basal levels in structures involved in higher processing of emotional stimuli (all nuclei of amygdala and hippocampi) in aged rats, as compared with young ones. No such increase in residual c-Fos expression was observed within the hypothalamus (except for the arcuate nuclei where one individual had higher basal c-Fos expression level). The former is in contrast to several studies showing no age-dependent differences in basal c-Fos expression $[5,13,28,34,36,42]$. This discrepancy might be explained by very recent results obtained by Knapska et al. [22]. They showed that structures involved in higher processing of emotional stimuli can be activated by between-subject transfer of information (for example by ultrasound vocalisation or odours [22]). In our experiment rats were housed in groups of two to three per cage and the cages were located close to each other in one experimental room. Control animals (kept in a separate cage, but still within the same room) were killed after being subjected to possible influence of signaling from animals returning from acute restraint test. According to Knapska et al. [22] such signaling can cause even greater neuronal activation within amygdala than the aversive stimulus itself. The fact that the high residual c-Fos expression was observed in the amygdala and hippocampi of aged animals could indicate that old rats might be more prone to excite following emotionally challenging stimuli. On the other hand, it might be a part of characteristics of Lewis rats strain resulting from deficient functioning of the HPA axis $[1,2,14,19]$. In order to elucidate this matter a comparison study on different strains of aged rats should be performed.

The general conclusion from this study is that regions of the fear/anxiety circuit (hypothalamus, amygdala and hippocampus) are differentially responsive to stress as a function of age in Lewis rats. The effect can be detected at both the magnitude and, to smaller extent, at the time-course of stress-evoked neuronal activation. Two basic groups of structures were identified: those directly responding to stress (HPA axis), which in old rats are released from inhibitory action of dorsomedial hypothalamic nuclei and therefore react with bigger neuronal activation in response to restraint with no concomitant changes in the temporal dynamics of the response, and the structures involved in higher processing of emotional cues, which functionally decrease with age. The two effects together might be a reason for higher rate of anxiety-related behaviors in OA rats.

\section{Acknowledgments}

The authors would like to thank Dr. Mark Hunt for his comments on the manuscript and Maciej Olszewski for the technical assistance. The study was supported by the Nencki Institute statutory grant to the Laboratory of the Limbic System.

\section{References}

[1] Armario A, Marti J. Forced swimming behavior is not related to the corticosterone levels achieved in the test: a study with four inbred strains. Physiol Behav 1996;59:369-73.
[2] Armario A, Gavalda A, Marti J. Comparison of the behavioural and endocrine response to forced swimming stress in five inbred strains of rats. Psychoneuroendocrinology 1995;20:879-90.

[3] Arnold FJL, De Lucas Bueno M, Shiers H, Hancock DC, Evan GI, Herbert J. Expressionof c-fos in regions of the basal limbic forebrain following intracerebroventricular corticotropin-releasing factor in unstressed and stressed male rats. Neuroscience 1992;51:377-90.

[4] Blokland A, Raajimakers W. Age-related changes in correlation between behavioral and biochemical parameters in Lewis rats. Behav Neural Biol 1993;60:52-61.

[5] Boguszewski P, Zagrodzka J. Expression of c-Fos in response to stressogenic stimuli in the amygdala of old vs. young rats - a preliminary study. Acta Neurobiol Exp 2005;65:191-4.

[6] Boguszewski P, Zagrodzka J. Emotional changes related to age in ratsbehavioral analysis. Behav Brain Res 2002;133:323-32.

[7] Bowers G, Cullinan WE, Herman JP. Region-specific regulation of glutamic acid decarboxylase (GAD) mRNA expression in central stress circuits. J Neurosci 1998;18(15):5938-47.

[8] Briski K, Gillen E. Differential distribution of Fos expression within the male rat preoptic area and hypothalamus in response tp physical vs. psychological stress. Brain Res Bull 2001;55:401-8.

[9] Cullinan WE, Herman JP, Battaglia DF, Akil H, Watson SJ. Pattern and time course of immediate early gene expression in rat brain following acute stress. Neuroscience 1995;64(2):477-505.

[10] Day HE, Badiani A, Uslaner JM, Oates MM, Vittoz NM, Robinson TE, et al. Environmental novelty differentially affects c-fos mRNA expression induced by amphetamine or cocaine in subregions of the bed nucleus of the stria terminalis and amygdale. J Neurosci 2001;21(2): 732-40.

[11] Day HE, Nebel S, Sasse S, Campeau S. Inhibition of the central extended amygdale by loud noise and restraint stress. Eur J Neurosci 2005;21:441-54.

[12] Dayas CV, Buller KM, Crane JW, Day TA. Stressor categorization: acute physical and psychological stressors elicit distinctive recruitment patterns in the amygdale and in medullary noradrenergic cell groups. Eur J Neurosci 2001;14(7):1143-52.

[13] Desjardins S, Mayo W, Valle M, Hancock M, Le Moal H, Simon H, et al. Effect of ageing on the basal expression of c-Fos, c-Jun and Egr-1 proteins in the hippocampus. Neurobiol Aging 1997;18(1):37-44.

[14] Dhabhar FS, McEwen BS, Spencer RL. Stress response, adrenal steroid receptor levels and corticosteroid-binding globulin levels-a comparison between Sprague-Dawley, Fischer 344 and Lewis rats. Brain Res 1993;616(1-2):89-98.

[15] Emmert MH, Herman JP. Differential forebrain c-fos mRNA induction by ether inhalation and novelty: evidence for distinctive stress pathways. Brain Res 1999;845:60-7.

[16] File SE, Seth P. A review of 25 years of the social interaction test. Eur J Pharmacol 2003;463(1-3):35-53.

[17] Frussa-Filho R, Otoboni JR, Gianotti AD, Amaral AC, Conceicao IM. Effect of age on antinociceptive effects of elevated plus-maze exposure. Braz J Med Biol Res 1992;25(8):827-9.

[18] Grizzle WE, Dallman MF, Schramm LP, Gann DS. Inhibitory and facilitatory hypothalamic areas mediating ACTH release in the cat. Endocrinology 1974;95(5):1450-61.

[19] Grota LJ, Bienen T, Felten DL. Corticosterone responses of adult Lewis and Fischer rats. J Neuroimmunol 1997;74:95-101.

[20] Herman JP, Tasker JG, Ziegler DR, Cullinan WE. Local circuit regulation of paraventricluar nucleus stress integration glutamate-GABA connections. Pharmacol Biochem Behav 2002;71:457-68.

[21] Honkaniemi J, Kainu T, Ceccatelli S, Rechardt L, Hokfelt T, Pelto-Huikko M. Fos and jun in rat central amygdaloid nucleus and paraventricular nucleus after stress. Neuroreport 1992;3(10):849-52.

[22] Knapska E, Nikolaev E, Boguszewski P, Walasek G, Blaszczyk J, Kaczmarek L, et al. Between-subject transfer of emotional information evokes specific pattern of amygdale activation. Proc Natl Acad Sci USA 2006;103(10):3858-62.

[23] Kovacs KJ. c-Fos as a transcription factor: a stressful (re)view from a functional map. Neurochem Int 1998;33(4):287-97. 
[24] Lamberty Y, Gower AJ. Age-related changes in spontaneous behavior and learning in NMRI mice from middle to old age. Physiol Behav 1992;51:81-8.

[25] Lamberty Y, Gower AJ. Spatial processing and emotionality in aged NMRI mice: a multivariate analysis. Physiol Behav 1993;54:339-43.

[26] Li JW, Watanabe M, Fujisawa Y, Shibuya T. Relation between agerelated changes in hyper-emotionality and serotonergic neuronal activitites in rat limbic system. Nihon Shinkei Seishin Yakurigaku Zasshi 1995;5: 231-8.

[27] Miyagawa H, Hasegawa M, Fukuta T, Amano M, Yamada K, Nabeshima T. Dissociation of impairment between spatial memory and motor functions and emotional behavior in aged rats. Behav Brain Res 1998;91: 73-81.

[28] Nagahara AH, Handa RJ. Age-related changes in c-fos mRNA induction after open-field exposure in the rat brain. Neurobiol Aging 1997;18(10):45-55.

[29] Nikolaev E, Kaczmarek L, Zhu SW, Winblad B, Mohammed AH. Environmental manipulation differentially alters c-Fos expression in amygdaloid nuclei following aversive conditioning. Brain Res 2002;957(1): 91-8.

[30] Pace TW, Gaylord R, Topczewski F, Girotti M, Rubin B, Spencer RL. Immediate-early gene induction in hippocampus and cortex as a result of novel experience is not directly related to the stressfulness of that experience. Eur J Neurosci 2005;22:1679-90.

[31] Pezzone MA, Lee W-S, Hoffman GE, Rabin BS. Induction of c-Fos immunoreactivity in the rat forebrain by conditioned and unconditioned aversive stimuli. Brain Res 1992;597:41-50.

[32] Ramos A, Mormede P. Stress and emotionality: a multidimensional and genetic approach. Neurosci Biobehav Rev 1998;22(1):33-57.
[33] Retchkiman I, Fisher B, Platt D, Wagner AP. Seizure induced C-Fos mRNA in the rat brain: comparison between young and aging animals. Neurobiol Aging 1996;17(1):41-4.

[34] Salchner P, Lubec G, Singewald N. Decreased social interaction in aged rats may not reflect changes in anxiety-related behaviour. Behav Brain Res 2004; $151: 1-8$.

[35] Savonenko A, Filipkowski RK, Werka T, Zielinski K, Kaczmarek L. Defensive conditioning-related functional heterogenity among nuclei of the rat amygdala revealed by c-Fos mapping. Neuroscience 1999;94(3):723-33.

[36] Schmoll H, Badan I, Fischer B, Wagner AP. Dynamics of gene expression for immediate early- and late genes after seizure activity in aged rats. Arch Gerontol Geriat 2001;32:199-218.

[37] Silveira MCL, Sandner G, Graeff FG. Induction of Fos immunoreactivity in the brain by exposure to the elevated plus-maze. Behav Brain Res 1993;56:115-8.

[38] Stohr T, Szuran T, Welzl H, Pliska V, Feldon J. Pryce CR Lewis/Fischer rat strain differences in endocrine and behavioural responses to environmental challenge. Pharmacol Biochem Behav 2000;67:809-19.

[39] Ter Horst GJ, Luiten PG. The projections of the dorsomedial hypothalamic nucleus in the rat. Brain Res Bull 1986;16(2):231-48.

[40] Touzani K, Marighetto A, Jaffard R. Fos imaging reveals ageing-related changes in hippocampal response to radial maze discrimination testing in mice. Eur J Neurosci 2003;17:628-40.

[41] Trneckova L, Armario A, Hynie S, Sida P, Klenerova V. Differences in the brain expression of c-fos mRNA after restraint stress in Lewis compared to Sprague-Dawley rats. Brain Res 2006;1077:7-15.

[42] Wagner AP, Schmoll H, Badan I, Platt D, Kessler C. Brain plasticity: to what extent do aged animals retain the capacity to coordinate gene activity in response to acute challenges. Exp Gerontol 2000;35:1211-27. 